\title{
EXPLOSIVE BREAKUP OF A WATER DROPLET WITH A NONTRANSPARENT SOLID INCLUSION HEATED IN A HIGH- TEMPERATURE GASEOUS MEDIUM
}

\author{
Margarita A. Dmitrienko, Maxim V. Piskunov, Pavel A. Strizhak, Anastasia A. Shcherbinina \\ National Research Tomsk Polytechnic University, 634050 Tomsk, Russia
}

\begin{abstract}
This paper investigates the evaporation of a water droplet with a comparably sized solid nontransparent inclusion in a high-temperature $(500-800 \mathrm{~K})$ gas medium. Water evaporates from the free surface of the inclusion. During this process, intensive vapor formation occurs on the inner interface "water droplet - solid inclusion" with the subsequent explosive decay of the droplet. Experiments have been conducted using highspeed (up to $10^{5} \mathrm{fps}$ ) video cameras "Phantom" and software "Phantom Camera Control". The conditions of the explosive vapor formation of the heterogeneous water droplet were found. The typical phase change mechanisms of the heterogeneous water droplet under the conditions of intensive heat exchange were determined.
\end{abstract}

\section{Introduction}

Nowadays, the development of modern chemical and energy technologies, that use heterogeneous and two-phase gas-vapor-droplet flows [1-3], is limited. This is mainly due to the insufficient [4] knowledge of the laws of phase changes under the conditions of the intensive heating (at gas temperature more than $500 \mathrm{~K}$ ) of heterogeneous droplets (with inclusions and admixtures).

Recent investigations [5-7] demonstrated that phase changes run more intensively in the presence of nontransparent non-metallic inclusions inside droplets. The studies [5-7] focused on the phase changes of homogeneous and heterogeneous water droplets and emulsions during their motion in a high-temperature gas medium.

The intensification of phase changes may occur not only on the free surface of a droplet, but also on the inner interface between two media - a solid particle and water. This hypothesis was formulated in studies [5-7] that describe the mechanism of heterogeneous droplet evaporation during its intensive heating.

Therefore, the experiments will be carried out to investigate the evaporation of a single droplet with a proportionate solid inclusion under conditions as in studies [5-7]. The results of experiments will help to substantiate the veracity of the hypothesis. One of the major problems is to determine the temperature of the external gas medium, when the explosive vapor formation occurs in a heterogeneous droplet on its inner interface between two media.

The aim of this work is to substantiate the feasibility of the several modes of evaporation: the evaporation of the heterogeneous water droplet from the free surface and intensive vapor formation on the inner interface between two media with explosive breakup of a droplet. 


\section{Experimental setup and research methods}

Fig. 1 shows our experimental setup. The evaporation of a heterogeneous droplet was continuously monitored by means of high-speed video recording. There were two high-speed (up to $10^{5} \mathrm{fps}$ ) video cameras 11 in our setup. The heterogeneous droplet was put on a ceramic rod 3 , and then inserted into a quartz cylinder 8 (the height of $1 \mathrm{~m}$, the inner and outer diameter of $0.2 \mathrm{~m}$ and $0.206 \mathrm{~m}$ ). A cylindrical channel 8 was filled with the combustion products obtained through the combustion of industrial alcohol. The processing of video recordings was performed with the help of specialized software in a personal computer 14 .
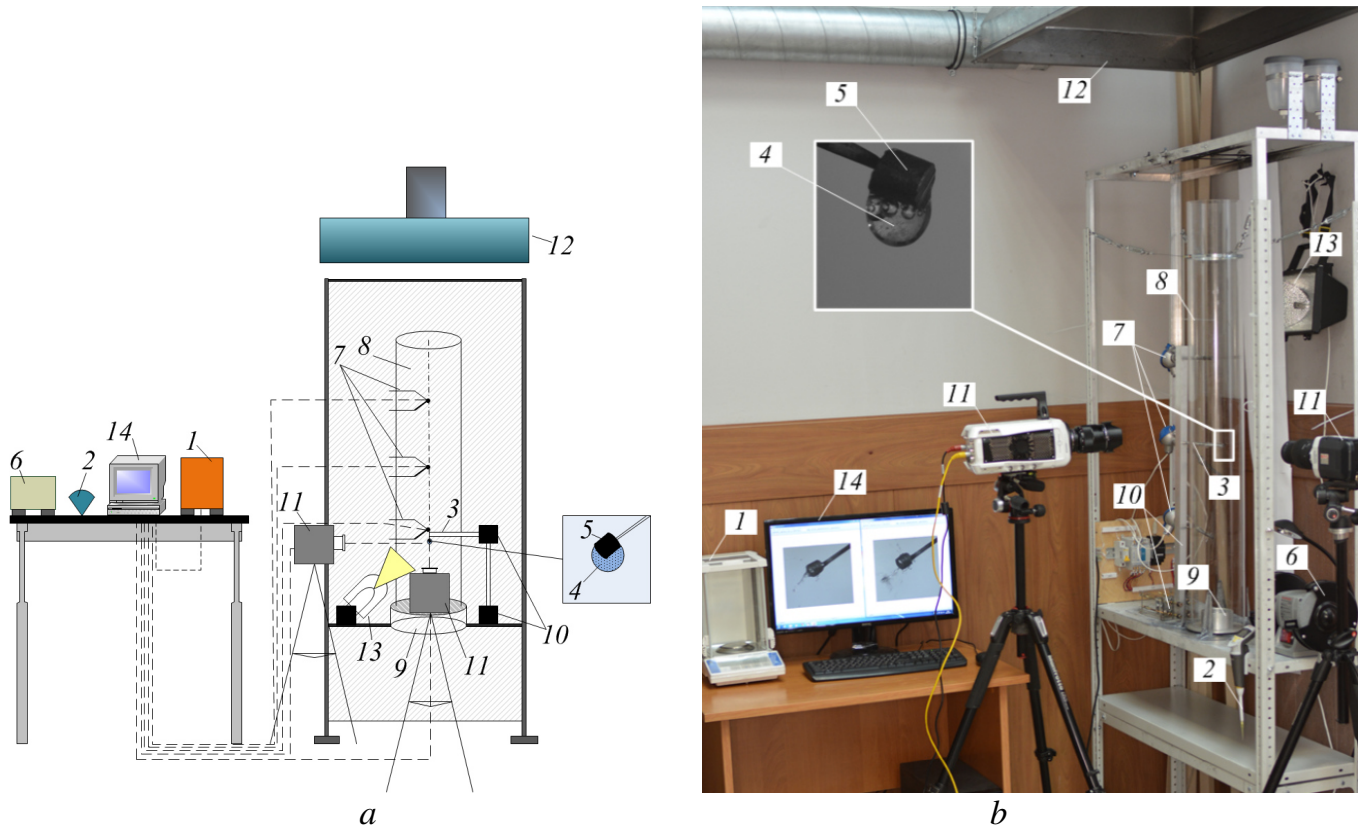

Figure 1. Scheme $(a)$ and physical configuration $(b)$ of an experimental setup: 1 - analytical balance, 2 - pipette, 3 - rod (holder for inclusion), 4 - droplet, 5 - inclusion, 6 - device for producing inclusions, 7 - thermocouples, 8 - quartz tube, 9 - burner, 10 - moving mechanisms, 11 - high-speed cameras, 12 - air flow system, 13 - spotlight, 14 - personal computer.

A specialized burner 9 was at the base of the cylindrical channel 8 . Industrial alcohol was poured into the burner. Industrial alcohol was used to generate high-temperature gases. There are three technological holes (at the height of $0.3 \mathrm{~m}, 0.5 \mathrm{~m}, 0.7 \mathrm{~m}$ relative to the base of the burner 9) in the cylindrical channel. The holes are made to fix chromel-alumel thermocouples 7 (the range of measured temperatures $273-1373 \mathrm{~K}$, measurement error $\pm 3.3 \mathrm{~K}$ ), as well as to insert the ceramic rod 3 with the heterogeneous droplet 4 into the channel (after measuring gas temperature).

Gas temperature varied from 500 to $800 \mathrm{~K}$ in the experiments. According to the developed technique, the rod 3 with a water droplet 4 and a solid graphite inclusion 5 was introduced into the cylindrical channel 8 through one of three holes. The cylindrical channel 8 was filled with combustion products obtained through the combustion of fuel (industrial alcohol) in the burner 9. An injection system 12 and special holes in the burner 9 were designed to maintain gas temperature within a certain constant value for 3 minutes with the deviation not more than $10 \mathrm{~K}$ (the absolute value was more than $500 \mathrm{~K}$ ). Thus, it was possible to conduct up to 5 experiments under identical heating conditions. A video recording lasted until complete water droplet evaporation or explosion (breakup or split) into several parts with substantially smaller sizes. The changes of droplet size and film thickness were recorded in each experiment. The characteristic times of heterogeneous droplet existence $\tau_{\mathrm{h}}$ were 
defined from recorded data using software "Phantom Camera Control". It was assumed that the characteristic time of heterogeneous droplet existence $\tau_{\mathrm{h}}$ is the period of heating droplet until its explosion or complete evaporation.

\section{Results and discussion}

The experiments demonstrate that the mechanism of phase changes during intensive heating can comply with one of three modes. The first vaporization mode is the evaporation of liquid only from the free surface of a droplet. Outside gas temperature is up to $600 \mathrm{~K}$.

The second mode has the following feature: the inclusion is fully immersed in the water droplet. The implementation of this mode depends on the thickness of the liquid film around the inclusion (more than $0.2 \mathrm{~mm}$ ), as well as on gas temperature (more than $700 \mathrm{~K}$ ). In turn, the second mode involves several stages: heating the liquid film around the inclusion, bubbles nucleation, the explosive breakup of the droplet. The explosive breakup of the droplet takes not more than 10 seconds. It lasts from the start of droplet heating until its destruction. The temperature varies from $700 \mathrm{~K}$ to $800 \mathrm{~K}$.

The third mode is characterized by the minimal time to achieve the conditions of explosive vapor formation. Gas temperature is more than $650 \mathrm{~K}$. It is important to note that the droplet increases (often even by several times relative to the initial droplet size) due to the growth of bubbles on the inner interface between two media.

Fig. 2 shows typical video frames of the heterogeneous water droplet corresponding to the established modes of vaporization.
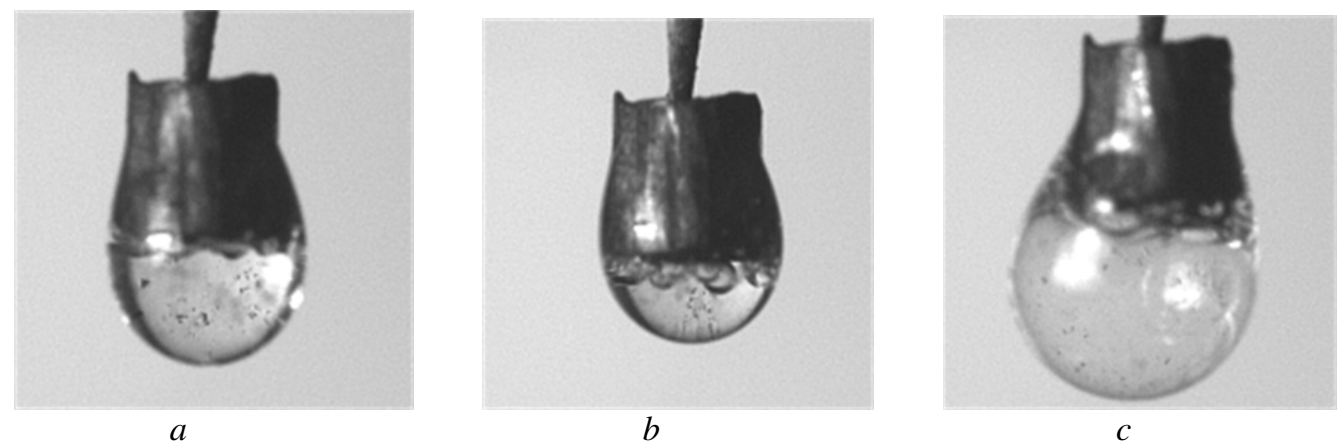

Figure 2. Typical video frames of heterogeneous water droplets corresponding to the established modes of vaporization: $a$ - the first mode (water evaporation from the free surface of a droplet); $b$ - the second mode (bubbles nucleation), $c$ - the third mode (increase of water droplet)

The present study proved that the probability of the explosive breakup of the droplet was minimal when the inclusion was multiple times lower than the droplet. Besides that, this conclusion follows from the analysis of previous reports [5-7]. This effect is more likely to occur when inclusions are commensurate with droplets (at least $50 \%$ of droplet volume) under conditions of high temperatures (over $650 \mathrm{~K}$ ).

The experiments allowed determining the impact features of characteristic inclusion sizes on the existence times (full evaporation from an outer surface or explosive vapor formation at the inner boundaries) of liquid droplets. First of all, the obtained essential nonlinearity of the determined dependences depends on the nonlinear dependence of evaporation rate on a temperature. The growth of the initial liquid mass only strengthens this nonlinearity. It was emphasized that with rising gas temperature the impact of characteristic inclusion sizes on liquid droplet existence times is strengthened. Such effect is due to an energy accumulation at the surface of an inclusion.

According to the experiments it can be expected that the presence of roughness on the inclusion surface and the certain position of a heterogeneous water droplet (inclusion edges are directed to the 
side of heat flow) assist the boiling intensification and the possible explosive vapor formation of water droplets.

It is worth noting that in this research the phase change mechanism is more intensive when the inclusion material (graphite) has the larger heat conductivity than water [8]. In this case, more energy passes through the inclusion and accumulates on the inner liquid - solid inclusion interface than in case of higher heat conductivity of water. It can be explained by total energy accumulating on the liquid - inclusion interface consists of the energy supplied by heat conductivity and the radiation energy implemented by the inclusion nontransparency $[9,10]$. Whereas in case of higher heat conductivity of water, the intensification of phase change at the inner interfaces is possible only by the accumulation of radiant heat flux. Thus, high heat conductivity of an inclusion material in comparison with water assists to increase a probability of explosive vapor formation and heterogeneous liquid droplet breakup.

The determined features allow drawing the conclusion that the optical and thermophysical properties of the inclusion and liquid will significantly influence on the conditions of the possible explosive vapor formation. Also, according to the experiments with different inclusions, it can be deduced that the intensification of explosive liquid droplet breakup is achieved by increasing the number of inclusion edges.

\section{Conclusion}

The novelty of this work is the established conditions of explosive breakup of a heterogeneous water droplet in a high-temperature gas medium. In this paper, we have defined three modes of phase change mechanisms of water droplets during intensive heating.

This work was financially supported by the grant of the President of the Russian Federation (MD2806.2015.8).

\section{References}

1. M.N. Nikitin, Industrial Power Engineering 12, 37 (2010) [in Russian].

2. P.A. Strizhak, J. Eng. Phys. Thermophys 86, 895 (2013).

3. A.Yu. Valdberg, K.P. Makeeva, Chem. Pet. Eng. 5, 42 (2010).

4. A.Yu. Varaksin, High Temp. 51, 377 (2013).

5. R.S. Volkov, G.V. Kuznetsov, P.A. Strizhak, Tech. Phys 59, 1770 (2014).

6. R.S. Volkov, G.V. Kuznetsov, P.A. Strizhak, Int. J. Therm. Sci. 88, 193 (2015).

7. O.V. Vysokomornaya, G.V. Kuznetsov, P.A. Strizhak, Fire Saf. J.. 70, 61 (2014).

8. N.B. Vargaftik, L.P. Filipov, A.A. Tarzimanov, E.E. Totskii, Handbook of Thermal Conductivity of Liquids and Gases (CRC Press, Inc., Boca Raton, 1994).

9. C. Kothandaraman, S. Subramanyan, Heat and Mass Transfer Data Book (Halsted Press/Wiley, Hoboken, New York, 1975).

10. H.Y. Wong, Handbook of Essential Formulae and Data on Heat Transfer for Engineers (Longman Group, United Kingdom, 1977). 